\title{
FAMILY PERIODIC PARALYSIS IN A MENTALLY DEFECTIVE BOY
}

\author{
BY \\ THOMAS A. COUSTON \\ From the Baldovan Institution, by Dundee \\ (RECEIVED FOR PUBLICATION JULY 26, 1954)
}

Family periodic paralysis is a condition characterized by periodic attacks of flaccid paralysis of the striated musculature accompanied by loss of tendon reflexes. Until recently the cause was unknown, but studies by Aitken, Allott, Castleden and Walker (1937) have correlated variations of the plasmo-serum potassium levels with the attacks of paralysis. In the case then studied the critical plasmo-serum potassium levels were considered to be $12 \mathrm{mg}$./100 ml., when marked muscular weakness occurred, and $10 \mathrm{mg} . / 100 \mathrm{ml}$., below which paralysis was observed. A fall in the plasmo-serum potassium level can be brought about in the normal person by large doses of glucose orally, the injection of insulin, or a combination of both. It appears that in susceptible subjects this reaction is much exaggerated. The condition was regarded as familial by Holtzapple (1905), who traced its incidence through several generations and considered transmission of the condition possible by either parent with an increased incidence of affected males. Although the disease may be traced through several generations, a generation may escape and the condition appear in the next. Holtzapple thought that family periodic paralysis may occur in association with a family history of migraine, and suggested that migraine might represent the equivalent of a paralytic attack. Only two post-mortem examinations appear to have been carried out, but nothing of significance was found in the nervous or muscular systems.

\section{Case Report}

The case recorded is that of a mentally defective boy, aged 16 when first observed. He is of imbecile grade with a residual left hemiplegia resulting from a birth injury, and is also subject to grand mal episodes. Following admission to the institution it was noted that his 'fits' fell into two distinct types: (1) typical grand mal attacks and (2) episodes of extreme flaccid paralysis of the limbs and trunk. Initially it was considered that the paralysis was a post-epileptic phenomenon. After careful observation, during which control of the grand mal episodes was established by the administration of phenytoin sodium $\left(1 \frac{1}{2}\right.$ gr. $(0 \cdot 1$ g.) t.i.d.), it was noted that the episodes of paralysis were unconnected with grand mal attacks and that paralysis mainly oocurred when the patient awoke in the morning. Eventually one paralytic episode was observed in its entirety.

Initially the patient became apprehensive and desired company; then he became elated and started singing. Swallowing movements and lip smacking were noted. His pupils became widely dilated and did not react to light. During this stage the patient was sitting up in bed moving his limbs normally. Speech became quite unintelligible and slurred, and his whole appearance suggested drunkenness. Shortly afterwards voluntary movements became much diminished, and he became distressed, carrying out rubbing and scratching movements over the left chest. Respiration became irregular, periods of air hunger alternating with rapid, shallow respirations. Gradually all movements of the limbs ceased, but the hands and feet did not appear to be affected, the right hand continuing the rubbing and scratching movements. During the fully developed paralytic stage the patient lay in bed, moving his hands and feet and speaking incoherently, but unable to raise his head from the pillow or his limbs from the mattress. Skin sensation was normal. Cutaneous and tendon reflexes were completely absent, though earlier in the attack an extensor plantar reflex was observed. The pulse rate fell to 58 , but heart action remained regular with no adventitious sounds. There was no cyanosis. The patient then, by hand signs and facial expression, showed an obvious desire to use a urinal, but although the desire to pass urine was obviously strong he was unable to do so. Skin temperature was low. The paralytic period continued for two and a half hours, during which the patient perspired freely, particularly in the head and face areas. He suddenly passed about $11 \mathrm{oz}$. (312 ml.) urine and appeared much brighter; his pupils returned to normal size and reacted to light. Within 15 minutes he was sitting up in bed and reaching for sweets on a window ledge near his bed.

In all, 24 such attacks have been observed over the past six months, the shortest lasting half an hour, the longest $\mathbf{4 8}$ hours. The pattern of all the attacks has been similar to that initially observed. It has also been noted that when the patient is confined to bed and sleeping 
during the daytime the attacks bear a relation to sleep, 19 having occurred on waking as opposed to five while the patient was ambulant.

During attacks all tendon and cutaneous reflexes are lost, and there is no muscular response to mechanical stimulation. Cutaneous sensation appears to be unaltered. The cranial nerves are unaffected. Plasmoserum potassium during the flaccid phase was estimated at $8.5 \mathrm{mg} . / 100 \mathrm{ml}$., the normal value in this patient being $17.5 \mathrm{mg} . / 100 \mathrm{ml}$. Urine examination revealed no abnormal constituents before or after an attack. Once the diagnosis was made treatment was instituted according to that carried out by Herrington (1937). At the start of the attack $10 \mathrm{~g}$. potassium citrate was given orally, followed by $5 \mathrm{~g}$. at 15-minute intervals. Recovery ensued within 20 minutes in all attacks when potassium citrate was administered. It was found empirically that a single dose of $10 \mathrm{~g}$. of potassium iodide produced the same result.

In order to establish a diagnosis it was decided to apply the findings of Aitkin et al. regarding the patient's reaction to oral glucose and the injection of insulin. Initially $50 \mathrm{~g}$. of glucose was given orally, and this dosage was repeated at half-hourly intervals. No paralytic episode occurred, but the boy exhibited the clinical signs of an impending attack, reaching the 'drunken' stage. The administration of 5 units of soluble insulin, however, precipitated a full paralytic episode within 30 minutes, flaccid paralysis lasting 20 minutes.

\section{Family History}

Investigation into the family history produced two significant points: (1) there was no history of any comparable condition on the paternal side of the family, but (2) the patient's mother admitted to having suffered from severe headaches since childhood, as did the maternal grandmother. Unfortunately the cooperation of the family was not good and no further information could be obtained. The parents stated that the patient had had 'jerking fits' since early childhood, but that from about the age of 12 he had two kinds of fits, 'jerking ones' and 'floppy ones', which they considered were 'both the same thing' and had paid no particular attention to them.

\section{Discussion}

This interesting case brings several important points to notice: first, the existence of family periodic paralysis in association with major epilepsy, the onset of periodic paralysis being at about 12 years of age, and, secondly, the absence of any comparable episode affecting any recent members of the family for at least two generations, but with a history of severe headaches, highly suggestive of migraine, on the maternal side of the family in two generations. Initially the diagnosis was uncertain, as the paralytic episodes were attributed to a postepileptic state until the epilepsy was controlled and a full paralytic episode was observed. The criteria for diagnosis were based on the findings of Aitken et al., namely, a plasmo-serum potassium level of under $10 \mathrm{mg}$. $/ 100 \mathrm{ml}$. during the paralytic phase, the loss of tendon and cutaneous reflexes with retention of cutaneous sensation, and the loss of muscle response to stimulation. This case fulfils all these requirements. The administration of glucose orally does not produce the full syndrome, but is sufficient to initiate the early clinical signs of an attack. Insulin, however, precipitates a characteristic paralytic episode. The question of sphincter control is difficult to understand, but in this case the sphincter appears to be in spasm and urine cannot be passed voluntarily. Further involvement of the autonomic nervous system is shown by the size of the pupils, fall of skin temperature, and perspiration. The relaxation of the urinary sphincter and the return of the pupils to normal precede recovery from the attack. Treatment as recommended by Herrington (1937) produces rapid recovery, but why a smaller dose of potassium iodide should have a similar effect to a larger dose of potassium citrate is uncertain. The marked frequency of attacks following sleep is not understood, but may be due to change in the blood chemistry during sleep.

\section{Summary}

A case of family periodic paralysis is reported occurring in an epileptic mentally defective patient. The onset of paralytic episodes occurred at about 12 years of age. During the paralytic episode the plasmo-serum potassium level falls below $10 \mathrm{mg}$./ $100 \mathrm{ml}$. to $8.5 \mathrm{mg} .100 \mathrm{ml}$. Flaccid paralysis of limbs and trunk occurs without the hands and feet being affected. There is no involvement of cranial nerves. Tendon and cutaneous reflexes are abolished during attacks, but cutaneous sensation is unimpaired. Muscular response to mechanical stimulation is lost. The autonomic nervous system is also involved, as demonstrated by the pupils, urinary sphincters, and skin. Rapid recovery follows the oral administration of either potassium citrate or iodide. The administration of glucose orally does not precipitate the full syndrome in this case, but the injection of insulin produces a paralytic episode. The incidence of attacks show a relation to sleep. There is no family history of family periodic paralysis as such, but there is a history highly suggestive of migraine in the patient's mother and maternal grandmother.

\section{REFERENCES}

Aitken, R. S. and Allott, E. N., Castleden, L. I. M. and Walker, M. (1937). Clin. Sci., 3, 47

Herrington, M. S. (1937). J. Amer. med. Ass., 108, 1339.

Holtzapple, G. E. (1905). Ibid., 45, 1224. 